\title{
Simulated-annealing studies of structural trends in carbon clusters
}

\author{
C. H. Xu, C. Z. Wang, C. T. Chan, and K. M. Ho \\ Ames Laboratory and Department of Physics and Microelectronics Research Center, Iowa State University, Ames, Iowa 50011
}

(Received 22 October 1992)

\begin{abstract}
The structural properties of carbon clusters $\left(\mathrm{C}_{n}, n=2-60\right)$ are studied using tight-binding moleculardynamics simulations. The low-energy ground-state configurations of the clusters are obtained by annealing and compressing $n$ carbon atoms $(n=2-60)$ from the gas phase at high temperatures ( $\geq 6000 \mathrm{~K})$. We observed a transition from one-dimensional linear and cyclic structures to two-dimensional cage structures as the number of atoms in the cluster increases beyond $n=20$.
\end{abstract}

Emerging as a new phase of carbon with a range of structural and chemical properties intermediate between small molecules and bulk phases, carbon clusters have received considerable experimental and theoretical attention over the past decade. ${ }^{1}$ Experimentally, most studies have been conducted with mass spectroscopy. ${ }^{2-6}$ Although the mass spectra provide useful information for understanding the relative stability of the clusters, they do not specify the geometries of the clusters. Recent breakthroughs in the synthesis process make it possible to produce $\mathrm{C}_{60}$ in sufficient purity and quantity to enable the identification of its structure as a truncated icosahedron. On the theoretical side, attempts have been made to determine the ground-state geometries of carbon clusters through total-energy calculations, ${ }^{1,7-11}$ and computer simulations. $^{12}$ However, up to now, reliable quantum chemical calculations are practical only for small clusters (up to about $n=10$ ) (Ref. 10) and are too time consuming for establishing a general structural trend. In this paper, we present a systematic study of the structure of carbon clusters as a function of cluster size using tightbinding molecular-dynamics (TBMD) simulations.

In our simulations, the interactions among the carbon atoms are described by a semiempirical tight-binding scheme $^{13}$ developed recently in our group. In this scheme, the total energy of the system, $E_{\text {tot }}$, is expressed as a quantum-mechanical bonding term, $E_{b s}$, plus a classical repulsive term, $E_{\text {rep }} . E_{b s}$, the sum of single-electron energies over all occupied electronic states, is determined by a set of tight-binding parameters, $E_{s}, E_{p}, V_{s s \sigma}, V_{s p \sigma}$, $V_{p p \sigma}$, and $V_{p p \pi}$, whose variations with interatomic separation $r$ are described by smooth short-ranged functions. $E_{\text {rep }}$ in our model is expressed as $\sum_{i} f\left(\sum_{j} \phi\left(r_{i j}\right)\right)$, with $\phi\left(r_{i j}\right)$ being a pairwise interaction between atoms $i$ and $j$, and $f$ a functional of $\sum_{j} \phi\left(r_{i j}\right)$. The parameters in such a TB total energy model are obtained by fitting $E_{\text {tot }}(r)$ to the results of first-principles local-density-functional calculations for the low-energy crystalline phases of carbon in the diamond, graphite, and linear chain structures. The energies and lattice constants of the highercoordinated metallic phases are also well reproduced. Our model describes well the phonons and elastic constants of the diamond and graphite crystals. Simulations of liquid carbon under various applied pressures reproduce accurately the results of $a b$ initio Car-Parrinello cal- culations, indicating that the potential has good transferability to a wide variety of environments.

The reliability of the potential for applications to carbon clusters is demonstrated by comparing the groundstate geometries obtained from our simulation with the results of accurate quantum-mechanical calculations for small clusters with $n \leq 11$. For every cluster of a given size, both the linear chain and the monocyclic ring structure are used as starting configuration for the simulations. After careful annealing, the clusters are found to converge to a unique final structure regardless of initial configuration. In the range $5 \leq n \leq 11$, we found that odd-numbered clusters prefer a linear structure, while even-numbered clusters prefer a ring structure. This result agrees well with the accurate $a b$ initio calculation results of Raghavachari and Binkley. ${ }^{10}$ For the very small clusters $(n \leq 4)$, the linear chain structure is found to be the most stable geometry.

For $n \geq 12$, the clusters can be trapped in local energy minima depending on the starting configuration. For these clusters, we perform the annealing simulation using the following procedures: First, the system is heated up to $10000 \mathrm{~K}$ for a few thousand time steps (one step is $\left.0.7 \times 10^{-15} \mathrm{~s}\right)$ to randomize their positions. At these temperatures, the carbon atoms are in the gas phase and have to be confined inside a hollow sphere of adjustable radius. Initially the radius of the hollow sphere is $2.5 R_{0}$, where $R_{0}$ is about the radius of the cluster at zero temperature. The atoms are subject to perfect reflections when they collide with the inner surface of the sphere. The system is then cooled down (in about 8000 steps) to $6000 \mathrm{~K}$, a temperature at which the clusters start to condense. At the same time, the radius of the sphere is reduced to $1.5 R_{0}$. While the temperature of the system is kept at $6000 \mathrm{~K}$, the radius of the sphere is further reduced to about $1.1 R_{0}$ at the slow rate of $0.008 R_{0}$ per thousand steps to facilitate the formation of the clusters. In general, we found that as the radius of the sphere is reduced, ring structures will first form and then change into the more stable cage structures upon further compression. Finally, the system is gradually cooled down to $T=0 \mathrm{~K}$ (at a cooling rate of $500 \mathrm{~K} / 1000$ steps) without any external constraint. For each cluster, the whole procedure takes about 50 ps of simulation time.

Our simulations show that monocyclic rings are ener- 
getically favorable for the clusters with $12 \leq n \leq 19$, while for clusters with $20 \leq n \leq 60$, the most stable structures are cages. Figure 1 shows some cage structures obtained by the simulation procedure above. For even-numbered clusters, the ground-state structures are closed cages while the odd-numbered cages are always found to contain some dangling bonds. For $n \geq 28$, the clusters obtianed from the simulations are not structurally perfect, but contain defects such as dangling bonds, fourmembered and seven-membered rings, or topological faults in the arrangement of the five-membered and sixmembered rings. The presence of such defects is probably due to the rapidity of the compression and cooling in the simulation since the larger clusters have more metastable states and require more annealing than smaller clusters. ${ }^{14,15}$

In Fig. 2, we plot the binding energy per atom as a function of cluster size ranging from $n=2$ to $n=70$ for the linear chain, monocyclic ring, graphitic fragment, and cage structures respectively. The energies of the ring structures for $20 \leq n \leq 43$ represent metastable intermediate structures obtained from our simulations. For graphitic fragments, we investigated clusters of $n=6,10,13$, $14,16,19,22,24,42$, and 54 , which have relatively high symmetries and lower number of dangling bonds within the range $6 \leq n \leq 60$. The calculations performed for these fragments are geometry optimized with all bond lengths constrained to be equal. Shown in Fig. 2 (open triangles) are also the energies of several "defect-free" cages $\left(\mathrm{C}_{28}, \mathrm{C}_{32}, \mathrm{C}_{60}, \mathrm{C}_{70}\right)$ obtained by relaxing the corresponding structures proposed by Kroto $^{16}$ based on geometry considerations. In particular, the relaxed $\mathrm{C}_{60}$ has been verified to be the ground-state geometry of $\mathrm{C}_{60}$ clusters.

We found that the ring structures are relatively more

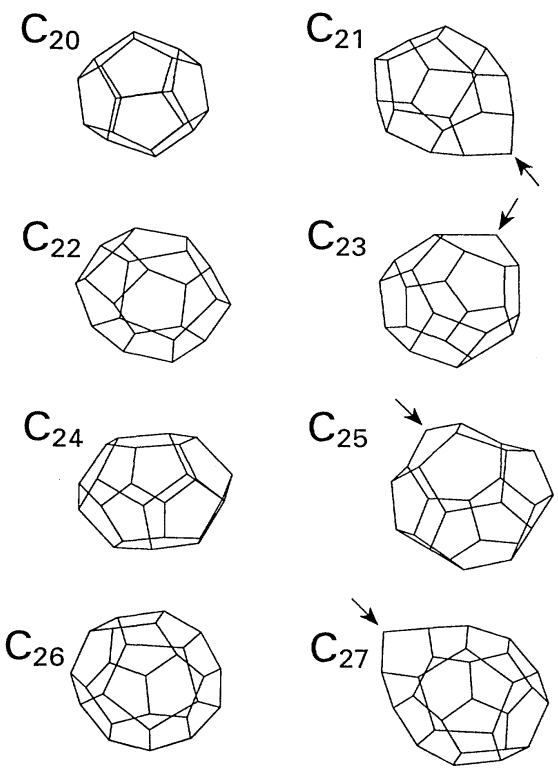

FIG. 1. Structures for $\mathrm{C}_{20}-\mathrm{C}_{27}$ at zero temperature. Arrows mark the position of dangling bonds. stable for cluster sizes with $n=4 m+2(m=1,2, \ldots)$. This trend is similar to the previous calculations of Hoffman ${ }^{7}$ and Bernholc and Phillips. ${ }^{9}$ The underlying physical origin of this behavior can be attributed to degeneracy of the $\pi$ electronic states of the carbon rings. ${ }^{9,10}$ For the cage structures, we found that the evennumbered clusters are energetically more favorable than the odd-numbered ones. This is because, geometrically, closed cage structures cannot be formed with all the atoms threefold coordinated when $n$ is odd. ${ }^{17}$

The most interesting feature in Fig. 2 is the transition from one-dimensional linear chain or ring structures to two-dimensional cage structures when $n$ reaches 20 . We found that $\mathrm{C}_{20}$ is the smallest closed cage that can be formed in our simulations. For $\mathrm{C}_{18}$ and $\mathrm{C}_{19}$ when the monocyclic rings are further compressed, metastable multiple ring structures (see Fig. 3) are formed instead of cages. As indicated in Fig. 2 these structures are less stable than the monocyclic rings.

Despite the presence of defects, the energies of the cages obtained by cooling and compressing from the gas phase are very close to that of the corresponding relaxed "defect-free" cages. The energy differences between the "cooked" and the relaxed cages are 0.015, 0.0, 0.094, and
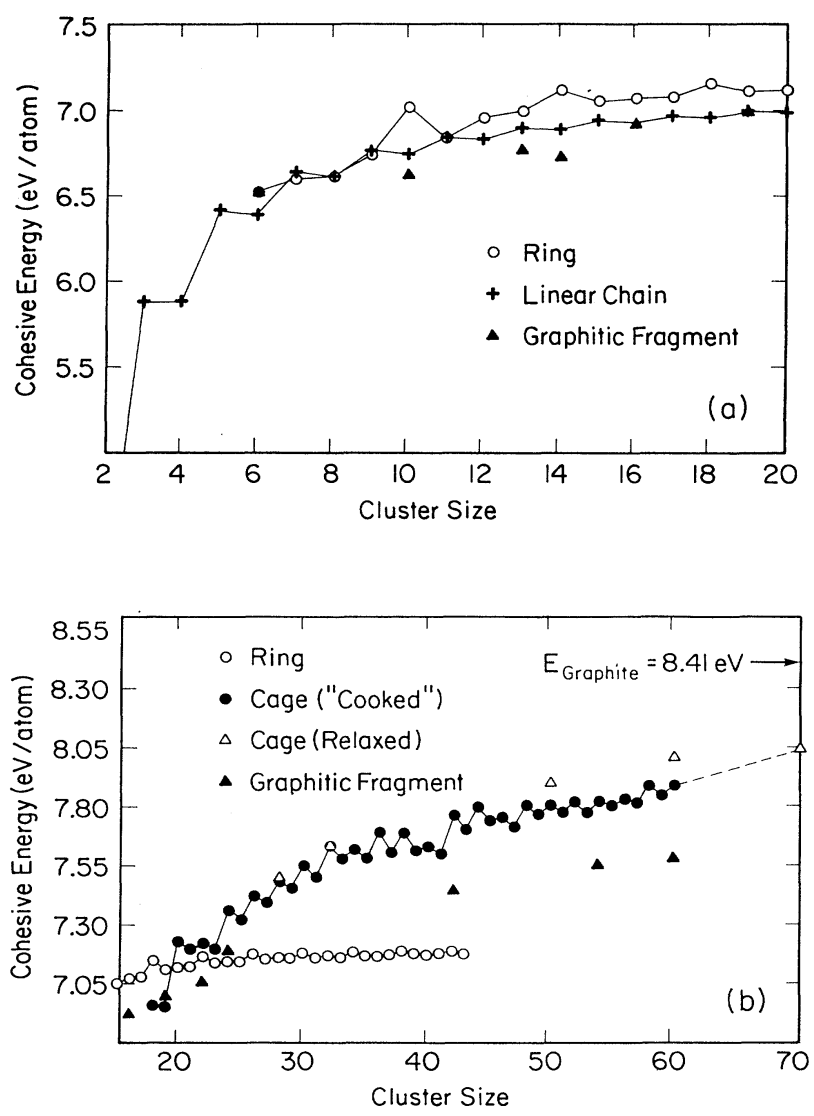

FIG. 2. Binding energy as a function of carbon cluster size for (a) chains, ring, and graphitic fragments with $2 \leq n \leq 20$, and (b) rings, cages, and graphitic fragments with $15 \leq n \leq 60$. See text for details. 
$0.119 \mathrm{eV} /$ atom, respectively, for $n=28,32,50$, and 60 . We believe that the general structural trend and transition obtained from the present simulation will remain the same even if more careful annealings are performed to eliminate the defects.

Finally, we note that the observed abundance of $\mathrm{C}_{60}$ molecules cannot be explained solely from its high formation energy. In fact, the formation energy of $\mathrm{C}_{70}$ is even higher than that of $\mathrm{C}_{60}$. We speculate that the abundance of $\mathrm{C}_{60}$ is related to its highly symmetric geometry which can lead to a higher survival probability in collisions after formation than other less symmetric clusters. We also note that the formation energy of the $\mathrm{C}_{60}$ and $\mathrm{C}_{70}$ is still far below $8.41 \mathrm{eV} /$ atom, the cohesive energy of the bulk graphite. This suggests that it is still possible to find even larger clusters with the two-dimensional cage geometry.

In summary, we have developed a tight-binding interatomic potential capable of describing accurately the structures and energetics of small carbon clusters $(n \leq 10)$ as well as the bulk crystalline phases of carbon. Using this potential we studied the structure and stability of

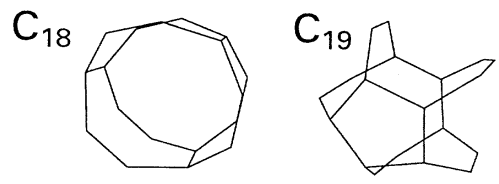

FIG. 3. Metastable multiple ring structures for $\mathrm{C}_{18}$ and $\mathrm{C}_{19}$ when the rings are compressed.

carbon clusters in the region of $2 \leq n \leq 60$. The study of larger clusters and the transition from cage structures to bulk phase are in progress.

Ames Laboratory is operated for the U.S. Department of Energy by Iowa State University under Contract No. W-7405-ENG-82. This investigation was supported by the Director for Energy Research, Office of Basic Energy Sciences including a grant of computer time on the Cray computers at Lawrence Livermore Laboratory and by NSF under Grant No. DMR-8819379.
${ }^{1}$ W. Weltner, Jr. and R. J. Van Zee, Chem. Rev. 89, 1713 (1989), and references therein.

${ }^{2}$ N. Furstenau and F. Hillenkamp, Int. J. Mass Spectrom. Ion. Phys. 37, 135 (1981).

${ }^{3}$ E. A. Rohlfing, D. M. Cox, and A. Kaldor, J. Chem. Phys. 81, 3322 (1984).

${ }^{4}$ H. W. Kroto, J. R. Heath, S. C. O'Brien, R. F. Curl, and R. E. Smalley, Nature (London) 318, 162 (1985).

${ }^{5}$ L. A. Bloomfield, M. E. Geusic, R. R. Freeman, and W. L. Brown, Chem. Phys. Lett. 121, 33 (1985).

${ }^{6}$ R. D. Knight, R. A. Walch, S. C. Foster, T. A. Miller, S. L. Mullen, and A. G. Marshall, Chem. Phys. Lett. 129, 331 (1986).

${ }^{7}$ R. Hoffmann, Tetrahedron 22, 521 (1966).

${ }^{8}$ K. S. Pitzer and E. Clementi, J. Am. Chem. Soc. 81, 4477 (1959).
${ }^{9}$ J. Bernholc and J. C. Phillips, J. Chem. Phys. 85, 2358 (1986).

${ }^{10}$ K. Raghavachari and J. S. Binkley, J. Chem. Phys. 87, 2191 (1987).

${ }^{11}$ W. Andreoni, D. Scharf, and P. Giannozzi, Chem. Phys. Lett. 173, 449 (1990).

12P. Ballone and P. Milani, Phys. Rev. B 42, 3201 (1990).

${ }^{13}$ C. H. Xu, C. Z. Wang, C. T. Chan, and K. M. Ho, J. Phys. Condens. Matter 4, 6047 (1992).

${ }^{14}$ R. O. Jones and O. Gunnarsson, Rev. Mod. Phys. 61, 689 (1989).

${ }^{15} \mathrm{H}$. W. Kroto and K. McKay, Nature (London) 331, 328 (1988).

${ }^{16}$ H. W. Kroto, Nature (London) 329, 529 (1987).

${ }^{17}$ T. G. Schmalz, W. A. Seitz, D. J. Klein, and G. E. Hite, J. Am. Chem. Soc. 110, 1113 (1988). 